\title{
Laboratory-confirmed respiratory infections as triggers for acute myocardial infarction and stroke: a self- controlled case series analysis of national linked datasets from Scotland
}

\author{
Charlotte Warren-Gash (10 ${ }^{1,2}$, Ruth Blackburn ${ }^{2}$, Heather Whitaker ${ }^{3}$, \\ Jim McMenamin ${ }^{4}$ and Andrew C. Hayward ${ }^{2,5}$
}

Affiliations: ${ }^{1}$ Faculty of Epidemiology and Population Health, London School of Hygiene and Tropical Medicine, London, UK. ${ }^{2}$ Institute of Health Informatics, University College London, London, UK. ${ }^{3}$ School of Mathematics and Statistics, Open University, Milton Keynes, UK. ${ }^{4}$ Health Protection Scotland, NHS National Services Scotland, Glasgow, UK. ${ }^{5}$ Institute of Epidemiology and Healthcare, University College London, London, UK.

Correspondence: Charlotte Warren-Gash, Room 248c, London School of Hygiene and Tropical Medicine, Keppel Street, London, WC1E 7HT, UK. E-mail: charlotte.warren-gash1alshtm.ac.uk

@ERSpublications

Laboratory-confirmed respiratory infections are linked to strokes and heart attacks in a Scottish population http://ow.ly/loOh30iyq0i

Cite this article as: Warren-Gash $\mathrm{C}$, Blackburn $\mathrm{R}$, Whitaker $\mathrm{H}$, et al. Laboratory-confirmed respiratory infections as triggers for acute myocardial infarction and stroke: a self-controlled case series analysis of national linked datasets from Scotland. Eur Respir J 2018; 51: 1701794 [https://doi.org/10.1183/ 13993003.01794-2017].

ABSTRACT While acute respiratory tract infections can trigger cardiovascular events, the differential effect of specific organisms is unknown. This is important to guide vaccine policy.

Using national infection surveillance data linked to the Scottish Morbidity Record, we identified adults with a first myocardial infarction or stroke from January 1, 2004 to December 31, 2014 and a record of laboratory-confirmed respiratory infection during this period. Using self-controlled case series analysis, we generated age- and season-adjusted incidence ratios (IRs) for myocardial infarction ( $\mathrm{n}=1227$ ) or stroke $(\mathrm{n}=762)$ after infections compared with baseline time.

We found substantially increased myocardial infarction rates in the week after Streptococcus pneumoniae and influenza virus infection: adjusted IRs for days 1-3 were 5.98 (95\% CI 2.47-14.4) and 9.80 (95\% CI 2.37-40.5), respectively. Rates of stroke after infection were similarly high and remained elevated to 28 days: day 1-3 adjusted IRs 12.3 (95\% CI 5.48-27.7) and 7.82 (95\% CI 1.07-56.9) for S. pneumoniae and influenza virus, respectively. Although other respiratory viruses were associated with raised point estimates for both outcomes, only the day 4-7 estimate for stroke reached statistical significance.

We showed a marked cardiovascular triggering effect of $S$. pneumoniae and influenza virus, which highlights the need for adequate pneumococcal and influenza vaccine uptake. Further research is needed into vascular effects of noninfluenza respiratory viruses.

This article has supplementary material available from erj.ersjournals.com

Received: Sept 012017 | Accepted after revision: Feb 132018

Copyright CERS 2018. This article is open access and distributed under the terms of the Creative Commons Attribution Licence 4.0. 


\section{Introduction}

Ischaemic heart disease, cerebrovascular disease and lower respiratory tract infections have been the three leading causes of death globally for $>15$ years $[1,2]$. Acute respiratory tract infections are associated with a short-term increase in the risk of acute cardiovascular events [3, 4]. A systematic review of observational studies showed that cardiovascular complications affected $\sim 18 \%$ of patients hospitalised for community-acquired pneumonia (CAP), with 5\% experiencing acute coronary syndrome [5]. As the population ages and the prevalence of multimorbidity increases, understanding and preventing interactions between diseases will become increasingly important.

In population-based studies using primary care electronic health records, acute respiratory tract infections are typically defined by clinical or syndromic criteria, as microbiological testing is rare. In hospital settings, laboratory testing for respiratory organisms is comparatively more common, but is still likely to be limited for patients with a cardiovascular, rather than respiratory, presentation. Despite extensive use of diagnostic tests, no causative organism is identified in $\sim 40 \%$ of hospitalised patients with CAP [6,7]. Inability to discriminate between different causative organisms therefore limits the utility of many existing studies for informing vaccine policy.

An alternative approach is to investigate whether existing vaccines against the two most common preventable respiratory organisms affecting older people, i.e. influenza virus and Streptococcus pneumoniae, reduce vascular risk. Meta-analysis of data from randomised controlled trials of influenza vaccine versus placebo in people with existing cardiovascular disease shows a $36 \%$ reduction in the risk of major adverse cardiovascular events (risk ratio 0.64, 95\% CI 0.48-0.86) associated with influenza vaccine [8]. For pneumococcal vaccine, data on cardiovascular outcomes from randomised controlled trials are lacking and selection biases associated with observational vaccine studies are well described [9]. Nevertheless, a recent systematic review and meta-analyses of case-control and cohort data suggested that receipt of pneumococcal vaccine was associated with a modest cardioprotective effect (pooled risk ratio 0.83, 95\% CI 0.71-0.97) from a random effects model [10], which was consistent with the pooled estimate from a similar systematic review [11].

In humans, both influenza virus and $S$. pneumoniae can have direct myocardial effects $[12,13]$ in addition to nonspecific pro-inflammatory and pro-coagulant changes, which are also seen in CAP and viral upper respiratory tract infections, and may exacerbate underlying atherosclerotic disease [14]. Understanding the relative contributions of different respiratory organisms to cardiovascular hospital admissions will inform health service planning and guide the development and targeting of interventions to reduce infection-related vascular risk.

Here, we aimed to quantify the association between laboratory-confirmed respiratory bacteria or virus infections and risk of first myocardial infarction or stroke using self-controlled case series analysis of anonymised linked electronic health records from Scotland in the UK. This study design is particularly suited to the investigation of transient exposures and acute outcomes, and has the major advantage of controlling implicitly for fixed between-person confounding [15], which may be otherwise difficult to account for using routinely collected health data.

\section{Methods}

Data sources

We used general/acute hospital inpatient data from the Scottish Morbidity Record (SMR01; www.ndc.scot. nhs.uk). This is a large national dataset containing details of all episodes of admitted patient care including day cases from acute specialities from hospitals in Scotland. Data are available from 1968 onwards with more than 1.4 million records are added each year. SMR01 records include sociodemographic details, clinical data on diagnoses and procedures undergone as well as administrative information such as admission and discharge dates.

We also obtained data on a range of laboratory-confirmed respiratory organisms from the Electronic Communication of Surveillance in Scotland (ECOSS; www.hps.scot.nhs.uk) dataset. ECOSS comprises information on all identifications of organisms or infections of clinical and public health significance

Conflict of interest: H. Whitaker has received grants from the MRC (methodology grant MR/L009005/1) and IMI/ EFPIA (ADVANCE), outside the submitted work.

Support statement: This study was funded by an Academy of Medical Sciences Starter Grant for Clinical Lecturers to C. Warren-Gash. C. Warren-Gash is supported by a Wellcome Intermediate Clinical Fellowship (201440_Z_16_Z). Funding information for this article has been deposited with the Crossref Funder Registry. 
reported from National Health Service laboratories throughout Scotland. Samples tested for respiratory organisms identified in ECOSS could originate from patients seeking medical attention for respiratory symptoms in either primary or secondary care settings. Extracts from these datasets were linked by the indexing team at National Services Scotland using a deterministic approach based on the CHI (Community Health Index) number (a unique patient identifier).

\section{Study design}

We conducted a self-controlled case series study to investigate the relative incidence of myocardial infarction or stroke occurring in time periods following laboratory-confirmed respiratory infections compared with baseline time periods for each individual. Derived from cohort logic, self-controlled case series is a design in which individuals act as their own controls during different time periods. This has the major advantage of eliminating time-invariant confounding $[15,16]$, which is particularly important for analyses involving routine health datasets in which potential confounding factors may not be adequately recorded. Self-controlled case series analysis also allows for the inclusion of time-varying confounders such as age and season in the models. Our null hypothesis was that exposure to acute respiratory organisms would not affect myocardial infarction and stroke incidence.

Self-controlled case series designs are statistically efficient relative to the cohort method [16]. Our sample size calculation, based on a relative incidence of 3 , a post-exposure risk period of 28 days and 10 years median follow-up duration, showed that 654 cases would be needed to estimate results with $90 \%$ power at the $5 \%$ significance level.

\section{Exposures and outcomes}

Records of all hospital admissions for first International Classification of Diseases-10-coded acute myocardial infarction (codes I21 and I23) and stroke (codes I60, I61 and I63) occurring in individuals aged $\geqslant 40$ years during the study period from January 1, 2004 to December 31, 2014 were obtained from the SMR01 dataset. We included a look-back period of 10 years to ensure that individuals did not have a myocardial infarction or stroke diagnosis before the study period. All linked ECOSS records were then extracted for specimens testing positive for one of the following respiratory organisms: influenza virus, parainfluenza virus, rhinovirus, respiratory syncytial virus, human metapneumovirus or S. pneumoniae during the same study period. Supplementary table S1 lists the specimen types and testing methods used for different respiratory organisms. For inclusion, a sample had to test positive by any one of the methods listed.

For each person, records of multiple respiratory organisms dated within 28 days of each other were de-duplicated within strata of virus or bacteria. For example, two virus records within 28 days were considered to represent a single episode of infection, with the onset date and diagnosis taken from the earliest record. For inclusion in our analysis, each individual required a record of first myocardial infarction or stroke and a record of at least one laboratory-confirmed respiratory infection (defined in supplementary table S1) during the study period. We restricted analyses to participants' first myocardial infarction or stroke, because experiencing a cardiovascular event may alter the risk of subsequent events and self-controlled case series is based upon the assumption that recurrent events occurring within individuals are independent.

\section{Statistical analysis}

We used conditional Poisson regression to calculate the relative incidence of first myocardial infarction and stroke occurring in pre-defined exposure periods after confirmed respiratory infections compared with unexposed or baseline time periods. We divided the exposure period into days 1-3, 4-7, 8-14 and 15-28 after acute respiratory tract infection (figure 1).

We defined a separate pre-exposure period of day $0-14$ before isolation of a respiratory organism and excluded this from baseline time: having a cardiovascular event in this period (e.g. a stroke) may affect the subsequent likelihood of recording of respiratory organisms (e.g. an episode of hospital-acquired pneumonia after stroke). We calculated the incidence ratio (IR) for myocardial infarction or stroke occurring in each exposure period compared with baseline time using conditional Poisson regression models that also adjusted for the time-varying confounders age in 5-year age groups and season in 3-month blocks.

We presented results separately for myocardial infarction and stroke and for respiratory bacteria (S. pneumoniae) and respiratory viruses (combined). In self-controlled case series analysis, the outcome should not censor follow-up so we conducted a sensitivity analysis to assess the effect of excluding records for individuals who died within 30 days of myocardial infarction or stroke [15]. We also investigated the effect of stratifying by age at cardiovascular event (age $<65$ versus $\geqslant 65$ years) and by whether respiratory 


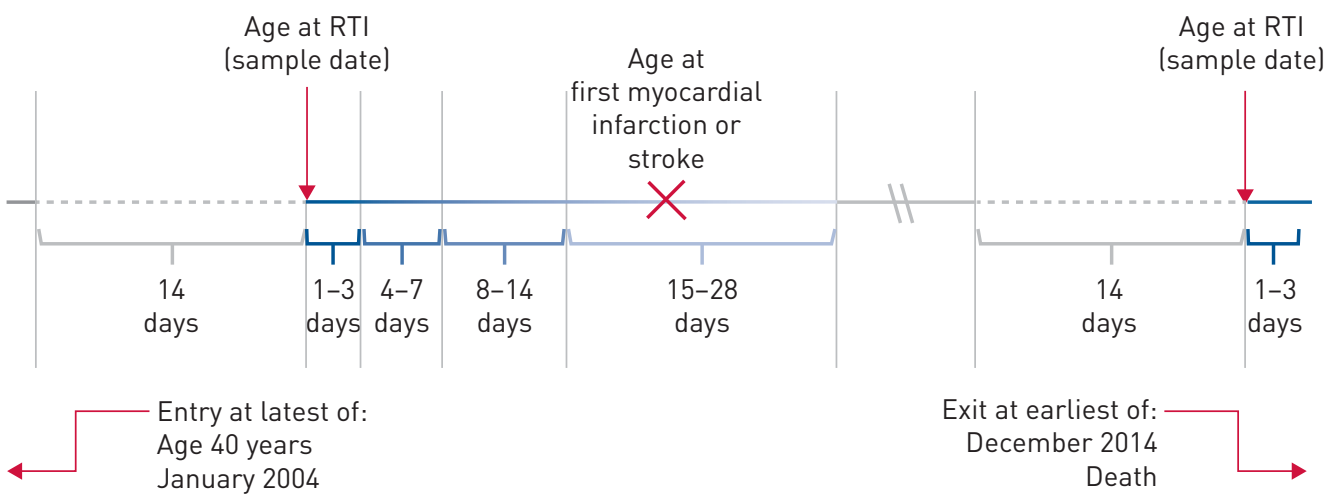

FIGURE 1 Example self-controlled case series timeline. RTI: respiratory tract infection. This timeline is based on age, and shows the risk period divided into $1-3,4-7,8-14$ and $25-28$ days after RTI. The grey solid line represents baseline time, the grey dashed line indicates excluded time, and the blue graduated line indicates risk periods and their proximity to the sample date. The incidence ratio for cardiovascular events occurring within each risk period compared with baseline time was calculated for each individual.

viruses were vaccine-preventable or not (influenza versus other respiratory viruses). Data were analysed using Stata version 14.0 (StataCorp, College Station, TX, USA).

\section{Ethics statement}

The study was approved by the Public Benefit and Privacy Panel for Health and Social Care, NHS Scotland (1516-0263) and the University College London Research Ethics Committee (3898/001).

\section{Results}

Description of study participants

We included 1227 individuals with first myocardial infarction (61\% male) and 762 with first stroke $(51 \%$ male) with a median (interquartile range (IQR)) age of 68 (59-77) years at the date of cardiovascular event. Patients with myocardial infarction had records for 924 separate episodes of S. pneumoniae infection and 491 episodes of respiratory virus infection during the study period (mean \pm SD $1.15 \pm 0.55$ samples per person). For stroke patients, the equivalent figures were 576 episodes of S. pneumoniae infection and 276 episodes of respiratory virus infection (mean \pm SD $1.12 \pm 0.45$ samples per person) (supplementary table S2). Table 1 describes the baseline characteristics of participants.

\section{Effect of respiratory organisms on first myocardial infarction and stroke}

The age- and season-adjusted IR for first myocardial infarction was markedly raised in the first 1-3 days after both respiratory bacterial and viral infections (IR for S. pneumoniae 5.98, 95\% CI 2.47-14.4; p $<0.001$ and IR for viruses 5.59, 95\% CI 1.77-17.6; $\mathrm{p}=0.003$ ), and persisted for $\sim 1$ week. For first episodes of stroke, the point estimate was even higher (adjusted IR for S. pneumoniae 12.3, 95\% CI 5.48-27.7; $\mathrm{p}<0.001$ and adjusted IR for viruses $6.79,95 \%$ CI 1.67-27.5; $\mathrm{p}=0.007$ ) for days $1-3$. Elevated rates of stroke following both bacterial and viral infections persisted to 28 days $(\mathrm{p}<0.001)$. Full results are shown in table 2.

\section{Sensitivity analysis removing fatal events}

Excluding 101 myocardial infarction records with a date of death within 30 days ( $8 \%$ of the total) made little difference to the overall findings: in this analysis, the age- and season-adjusted IR for myocardial infarction associated with S. pneumoniae was 5.41 (95\% CI 2.02-14.5) in days 1-3; for respiratory viruses, the equivalent adjusted IR was 6.20 (95\% CI 1.97-19.6). Patterns across the 1-28-day risk period were similar to the main model. For stroke, 107 records of fatal events (14\% of the total) were excluded. After this, the new adjusted IR associated with S. pneumoniae was 9.87 (95\% CI 3.67-26.5) in days 1-3 and all IRs remained elevated to 28 days. In contrast, for respiratory viruses, although all point estimates were elevated, only the day $8-14$ and 15-28 estimates reached statistical significance. Table 3 shows the full results of this sensitivity analysis.

\section{Stratified analysis}

By organism (influenza versus all other respiratory viruses)

In general, influenza virus was associated with higher IRs for both myocardial infarction and stroke than all other respiratory viruses combined, although numbers in some cells were small and confidence intervals overlapped. For myocardial infarction, the adjusted day 1-3 IR for influenza virus was 9.80 
TABLE 1 Baseline characteristics of participants

\begin{tabular}{|c|c|c|}
\hline & Myocardial infarction & Stroke \\
\hline Subjects & 1227 & 762 \\
\hline Records of respiratory organism ${ }^{\#}$ & 1415 & 852 \\
\hline Streptococcus pneumoniae & 924 (65.3) & $576(67.6)$ \\
\hline Influenza virus & $179(12.7)$ & $114(13.4)$ \\
\hline Rhinovirus & $122(8.6)$ & 79 (9.3) \\
\hline Parainfluenza virus & $69(4.9)$ & $34(4.0)$ \\
\hline Respiratory syncytial virus & $69(4.9)$ & $24(2.8)$ \\
\hline Human metapneumovirus & $52(3.7)$ & $25(2.9)$ \\
\hline \multicolumn{3}{|l|}{ Age at first CVD event years } \\
\hline $40-49$ & $120(9.8)$ & $69(9.1)$ \\
\hline $50-59$ & $219(17.8)$ & $136(17.8)$ \\
\hline $60-69$ & 347 (28.3) & $188(24.7)$ \\
\hline $70-79$ & $363(29.6)$ & 220 (28.9) \\
\hline $80-89$ & $161(13.1)$ & $131(17.2)$ \\
\hline$\geqslant 90$ & $17(1.4)$ & $18(2.4)$ \\
\hline \multicolumn{3}{|l|}{ Sex } \\
\hline Male & $751(61.2)$ & $392(51.4)$ \\
\hline Female & 476 (38.8) & $370(48.6)$ \\
\hline \multicolumn{3}{|c|}{ Length of stay associated with CVD event days } \\
\hline Median & 5 & 11 \\
\hline Lower quartile & 3 & 4 \\
\hline Upper quartile & 10 & 41 \\
\hline \multicolumn{3}{|l|}{ Died $<30$ days after CVD event } \\
\hline Yes & $101(8.2)$ & $107(14.0)$ \\
\hline No & 1227 (91.8) & $655(86.0)$ \\
\hline \multicolumn{3}{|l|}{ Died within study period } \\
\hline Yes & $473(38.5)$ & $345(45.3)$ \\
\hline No & $754(61.5)$ & $417(54.7)$ \\
\hline \multicolumn{3}{|c|}{$\begin{array}{l}\text { Data are presented as } \mathrm{n} \text { or } \mathrm{n}(\%) \text {. CVD: cardiovascular disease. \#: for } S \text {. pneumoniae, the number of } \\
\text { episodes recorded per person across the whole study period for both myocardial infarction and stroke } \\
\text { patients ranged from } 1 \text { to } 8 \text {; for respiratory viruses, the respective numbers of episodes recorded per } \\
\text { person ranged from } 1 \text { to } 3 \text { (influenza virus), } 1 \text { to } 3 \text { (rhinovirus), } 1 \text { to } 4 \text { (parainfluenza virus), } 1 \text { to } 2 \\
\text { (respiratory syncytial virus) and } 1 \text { (human metapneumovirus). }\end{array}$} \\
\hline
\end{tabular}

TABLE 2 Age- and season-adjusted incidence ratio (IR) for first myocardial infarction and first stroke in periods after Streptococcus pneumoniae and respiratory viruses (combined) compared with baseline time

\begin{tabular}{|c|c|c|c|c|}
\hline Time period after sample days & $\begin{array}{l}\text { IR for S. pneumoniae } \\
\qquad(95 \% \mathrm{CI})\end{array}$ & p-value & $\begin{array}{l}\text { IR for respiratory } \\
\text { viruses }(95 \% \mathrm{CI})\end{array}$ & p-value \\
\hline \multicolumn{5}{|l|}{ Outcome: myocardial infarction" } \\
\hline $1-3$ & $5.98(2.47-14.4)$ & $<0.001$ & $5.59(1.77-17.6)$ & 0.003 \\
\hline $4-7$ & $3.79(1.41-10.1)$ & 0.008 & $3.00(0.74-12.1)$ & 0.12 \\
\hline $8-14$ & $1.65(0.53-5.15)$ & 0.38 & $1.00(0.14-7.15)$ & 0.99 \\
\hline $15-28$ & $2.04(0.96-4.31)$ & 0.06 & $2.12(0.79-5.70)$ & 0.13 \\
\hline Baseline & 1.00 & & 1.00 & \\
\hline \multicolumn{5}{|l|}{ Outcome: stroke } \\
\hline $1-3$ & $12.3(5.48-27.7)$ & $<0.001$ & $6.79(1.67-27.5)$ & 0.007 \\
\hline $4-7$ & $8.23(3.39-19.9)$ & $<0.001$ & $5.43(1.34-21.9)$ & $<0.001$ \\
\hline $8-14$ & $4.90(2.02-11.8)$ & $<0.001$ & $5.01(1.59-15.7)$ & $<0.001$ \\
\hline $15-28$ & $4.09(2.02-8.27)$ & $<0.001$ & $4.02(1.62-9.95)$ & $<0.001$ \\
\hline Baseline & 1.00 & & 1.00 & \\
\hline
\end{tabular}

\#: $n=1227 ;{ }^{9}: \mathrm{n}=762$. 
TABLE 3 Age- and season-adjusted incidence ratio (IR) for first myocardial infarction with $\geqslant 30$ day survival and first stroke with $\geqslant 30$ day survival in periods after Streptococcus pneumoniae and respiratory viruses (combined) compared with baseline time

\begin{tabular}{|c|c|c|c|c|}
\hline Time period after sample days & $\begin{array}{l}\text { IR for S. pneumoniae } \\
\qquad(95 \% \mathrm{CI})\end{array}$ & p-value & $\begin{array}{l}\text { IR for respiratory } \\
\text { viruses }(95 \% \mathrm{CI})\end{array}$ & p-value \\
\hline \multicolumn{5}{|c|}{ Outcome: nonfatal myocardial infarction ${ }^{\#}$} \\
\hline $1-3$ & $5.41(2.02-14.5)$ & 0.001 & $6.20(1.97-19.6)$ & 0.002 \\
\hline $4-7$ & $3.13(1.01-9.75)$ & 0.049 & $3.25(0.80-13.1)$ & 0.10 \\
\hline $8-14$ & $1.78(0.57-5.56)$ & 0.32 & $1.06(0.15-7.53)$ & 0.96 \\
\hline $15-28$ & $2.19(1.04-4.62)$ & 0.04 & $2.23(0.83-5.98)$ & 0.11 \\
\hline Baseline & 1.00 & & 1.00 & \\
\hline \multicolumn{5}{|l|}{ Outcome: nonfatal stroke ${ }^{\pi}$} \\
\hline $1-3$ & $9.87(3.67-26.5)$ & $<0.001$ & $3.97(0.55-28.5)$ & 0.17 \\
\hline $4-7$ & $7.60(2.83-20.4)$ & $<0.001$ & $3.15(0.44-22.6)$ & 0.25 \\
\hline $8-14$ & $5.34(2.20-13.0)$ & $<0.001$ & $5.74(1.82-18.1)$ & 0.003 \\
\hline $15-28$ & $3.90(1.84-8.27)$ & $<0.001$ & $3.70(1.35-10.1)$ & 0.01 \\
\hline Baseline & 1.00 & & 1.00 & \\
\hline
\end{tabular}

${ }^{\#}: \mathrm{n}=1227 ;{ }^{\text {ๆ: }} \mathrm{n}=655$.

(95\% CI 2.37-40.5) and for other respiratory viruses it was 2.81 (95\% CI 0.39-20.3). For stroke, the adjusted day 1-3 IR after influenza virus was 7.82 (95\% CI 1.07-56.9) and for other respiratory viruses it was 4.86 (95\% CI 0.67-35.4). IRs for other time periods are shown in table 4.

By age

Stratifying by age at cardiovascular event was also hampered by small numbers and some cells contained zero events. Nevertheless, those aged $<65$ years tended to have higher adjusted IRs for myocardial infarction and stroke after respiratory bacteria and virus infections than those aged $\geqslant 65$ years, although there were overlapping confidence intervals. Full results are shown in table 5.

\section{Discussion}

We show that infection with S. pneumoniae and a range of different respiratory viruses is associated with increased rates of first myocardial infarction and stroke in a Scottish adult population. The period of elevated risk associated with infections persisted to 28 days for stroke, but was more transient for myocardial infarction, suggesting potentially different mechanisms. While the vaccine-preventable organisms influenza virus and S. pneumoniae were associated with particularly high IRs for vascular

TABLE 4 Age- and season-adjusted incidence ratio (IR) for first myocardial infarction and first stroke after influenza and all other respiratory viruses (combined) compared with baseline time periods

\begin{tabular}{|c|c|c|c|c|}
\hline Time period after sample days & $\begin{array}{l}\text { IR for influenza virus } \\
\qquad(95 \% \mathrm{CI})\end{array}$ & p-value & $\begin{array}{l}\text { IR for other respiratory } \\
\text { viruses }(95 \% \mathrm{CI})\end{array}$ & p-value \\
\hline \multicolumn{5}{|l|}{ Outcome: myocardial infarction ${ }^{\#}$} \\
\hline $1-3$ & $9.80(2.37-40.5)$ & 0.002 & $2.81(0.39-20.3)$ & 0.31 \\
\hline $4-7$ & $3.98(0.55-28.9)$ & 0.17 & $2.30(0.32-16.6)$ & 0.41 \\
\hline $8-14$ & $2.72(0.38-19.5)$ & 0.32 & $\sim 0$ & 0.98 \\
\hline $15-28$ & $2.77(0.68-11.2)$ & 0.15 & $1.58(0.39-6.41)$ & 0.64 \\
\hline Baseline & 1.00 & & 1.00 & \\
\hline \multicolumn{5}{|l|}{ Outcome: stroke ${ }^{\pi}$} \\
\hline $1-3$ & $7.82(1.07-56.9)$ & 0.042 & $4.86(0.67-35.4)$ & 0.12 \\
\hline $4-7$ & $\sim 0$ & 0.99 & $8.72(2.14-35.6)$ & 0.003 \\
\hline $8-14$ & $8.13(1.98-33.3)$ & 0.004 & $2.35(0.32-17.0)$ & 0.40 \\
\hline $15-28$ & $5.13(1.55-17.0)$ & 0.007 & $2.59(0.63-10.6)$ & 0.19 \\
\hline Baseline & 1.00 & & 1.00 & \\
\hline
\end{tabular}

\#: $n=1227 ;$ १: $n=762$. 
TABLE 5 Age- and season-adjusted incidence ratio (IR) for first myocardial infarction and first stroke stratified by age $<65$ and $\geqslant 65$ years in periods after Streptococcus pneumoniae and respiratory viruses (combined) compared with baseline time

\begin{tabular}{|c|c|c|c|c|}
\hline Time period after sample days & $\begin{array}{l}\text { IR for S. pneumoniae } \\
\qquad(95 \% \mathrm{CI})\end{array}$ & p-value & $\begin{array}{l}\text { IR for respiratory } \\
\text { viruses }(95 \% \mathrm{CI})\end{array}$ & p-value \\
\hline \multicolumn{5}{|l|}{$\begin{array}{l}\text { Outcome: myocardial infarction } \\
\text { aged }<65 \text { years }^{\#}\end{array}$} \\
\hline $1-3$ & $7.97(1.97-32.2)$ & 0.004 & $16.1(5.12-50.9)$ & $<0.001$ \\
\hline $4-7$ & $12.7(4.69-34.4)$ & $<0.001$ & $4.20(0.59-30.1)$ & 0.15 \\
\hline $8-14$ & $1.77(0.25-12.7)$ & 0.572 & $\sim 0$ & 0.997 \\
\hline $15-28$ & $1.93(0.48-7.81)$ & 0.355 & $4.08(1.29-12.9)$ & 0.017 \\
\hline Baseline & 1.00 & & 1.00 & \\
\hline \multicolumn{5}{|l|}{$\begin{array}{l}\text { Outcome: myocardial infarction } \\
\text { aged } \geqslant 65 \text { years }\end{array}$} \\
\hline $1-3$ & $5.16(1.65-16.1)$ & 0.005 & $\sim 0$ & 0.998 \\
\hline $4-7$ & $\sim 0$ & 0.998 & $2.35(0.33-17.0)$ & 0.40 \\
\hline $8-14$ & $1.60(0.40-6.45)$ & 0.51 & $1.57(0.22-11.2)$ & 0.65 \\
\hline $15-28$ & $2.09(0.86-5.06)$ & 0.10 & $0.82(0.11-5.9)$ & 0.85 \\
\hline Baseline & 1.00 & & 1.00 & \\
\hline \multicolumn{5}{|l|}{ Outcome: stroke aged $<65$ years $^{+}$} \\
\hline $1-3$ & $37.4(13.7-102)$ & $<0.001$ & $23.4(5.71-96.3)$ & $<0.001$ \\
\hline $4-7$ & $7.83(1.09-56.4)$ & 0.041 & $\sim 0$ & 0.998 \\
\hline $8-14$ & $14.2(4.48-45.2)$ & $<0.001$ & $10.5(2.55-42.9)$ & 0.001 \\
\hline $15-28$ & $9.74(3.56-26.7)$ & $<0.001$ & $5.90(1.68-20.7)$ & 0.006 \\
\hline Baseline & 1.00 & & 1.00 & \\
\hline \multicolumn{5}{|l|}{ Outcome: stroke aged $\geqslant 65$ years ${ }^{\S}$} \\
\hline $1-3$ & $5.21(1.29-21.0)$ & 0.02 & $\sim 0$ & 0.999 \\
\hline $4-7$ & $7.69(2.84-20.8)$ & $<0.001$ & $6.83(1.66-28.1)$ & 0.008 \\
\hline $8-14$ & $2.35(0.58-9.48)$ & 0.23 & $2.33(0.32-16.8)$ & 0.40 \\
\hline $15-28$ & $2.45(0.91-6.60)$ & 0.08 & $2.52(0.62-10.3)$ & 0.20 \\
\hline Baseline & 1.00 & & 1.00 & \\
\hline
\end{tabular}

${ }^{\#}: n=498 ;{ }^{\text {ๆ }}: n=729 ;{ }^{+}: n=301 ;{ }^{\S}: n=461$.

events, we also showed an effect of other viruses, for which vaccines are not currently available, although this was significant only for stroke. The finding of higher IRs in people aged $<65$ years warrants further exploration but may relate to vaccine: influenza vaccine uptake reached $77 \%$ in Scotland during the period of observation among those aged $\geqslant 65$ years [17] but was much lower in younger people, who may not be eligible for vaccination. This highlights the need to consider other prevention and treatment options for acute respiratory tract infections, especially among patients at high risk of cardiovascular disease.

Strengths of our study include the novel use of data on laboratory-confirmed respiratory infections from 11 years of national surveillance in Scotland linked to robust vascular outcome measures from national hospitalisation records. A unique advantage of the self-controlled case series method is its ability to control for fixed confounders [15], which may distort findings from other similar studies using routine health data. We also controlled for the time-varying effects of age and season, so residual confounding by other time-varying factors is unlikely. We showed a biological gradient of stroke and, to a lesser extent, myocardial infarction risk after acute respiratory tract infections, which is likely to indicate a causal relationship. Use of laboratory-confirmed infection measures improved diagnostic specificity and therefore the relevance of results for vaccine policy. Our population-based approach means that results should be generalisable to similar Northern European populations.

Our study nevertheless had some limitations. Although primary analyses were well powered and self-controlled case series is an efficient design, the relatively small population size in Scotland meant that the study lacked power for some subgroup analyses. It was not possible to investigate the effects of individual noninfluenza respiratory viruses on vascular events or to examine age effects in detail. We were also unable to investigate the effect of co-infections separately, although the number of patients experiencing co-infections was extremely small. During the study period there were changes in microbiological testing practices, e.g. PCR was not widely used in 2004. However, if testing methods were relatively less sensitive in the earlier years of our study, this would potentially reduce the number of patients meeting inclusion criteria (and therefore statistical power) rather than introduce bias. While we 
could not exclude the chance that some organisms isolated might represent incidental colonisation rather than symptomatic infections, this would have the effect of biasing results towards the null. In addition, we used the day after the date of respiratory sampling as the start of the risk period. However, the infection onset date would have occurred earlier, most likely during the 14-day period excluded from baseline time, which would have led to underestimation of the true effect size.

The effect of influenza virus on cardiovascular hospital admissions and deaths is well documented in different settings $[18,19]$. Recent studies describe high rates of morbidity and seasonal mortality among older adults infected with other respiratory viruses, including human metapneumovirus, parainfluenza virus, rhinovirus and respiratory syncytial virus [20-23]. We recently showed that circulation of adenovirus, human metapneumovirus, influenza virus, rhinovirus and respiratory syncytial virus was associated with hospitalisations for cardiovascular disease at the population level [24]. In this self-controlled case series analysis, we confirm and extend findings from individual-level studies using primary care data that show an acute triggering effect of clinically diagnosed acute respiratory tract infections on myocardial infarction and stroke $[25,26]$. The higher point estimates for influenza compared with other respiratory viruses are consistent with previous findings suggesting that cardiovascular complications are more frequent with influenza than other viruses such as rhinovirus among hospitalised adults [20]

Our findings that both influenza virus and S. pneumoniae have specific triggering effects on myocardial infarction and stroke emphasise the need to encourage uptake of influenza and pneumococcal vaccines wherever indicated, especially among populations with existing heart disease in whom influenza vaccine uptake is suboptimal [27]. Trials are ongoing to investigate the effectiveness of pneumococcal vaccine against cardiovascular events [28], and the effects of different formulations and target populations for influenza vaccination on individual cardiovascular end-points (ClinicalTrials.gov identifiers NCT02831608, NCT02762851, NCT02787044 and NCT02268500). Demonstration of significant cardiovascular protection will inform updated cost-effectiveness analyses and consideration of extending indication for these vaccines to at-risk groups who do not meet current guidelines for vaccination.

The finding that other respiratory viruses for which vaccines are not available also act as cardiovascular triggers merits further exploration. It potentially strengthens the case for considering antithrombotic strategies during acute respiratory tract infections for vulnerable groups, especially as recent evidence points to a dual effect of acute respiratory tract infections and symptomatic nonsteroidal anti-inflammatory drug treatment on cardiovascular risk [29]. Future research should focus on informing development and delivery of stratified interventions to reduce vascular risk associated with a range of respiratory organisms. Large robust population studies are needed to investigate the effects of acute respiratory tract infections on vascular risk in different population subgroups, e.g. those with individual cardiovascular risk factors, and for subsets of infections such as bacteria causing atypical pneumonias and, in particular, noninfluenza respiratory viruses.

In conclusion, we show a marked cardiovascular triggering effect of $S$. pneumoniae and influenza virus, as well as raised point estimates for myocardial infarction and stroke associated with other confirmed respiratory viruses.

\section{Acknowledgements}

We thank Doug Kidd of the electronic Data Research and Innovation Service (eDRIS) at National Services Scotland (Edinburgh, UK) for assistance with study design, approvals and data access.

Author contributions: All authors contributed to design of the study and/or analyses, interpretation of data, and critical revision of the manuscript for important intellectual content. R. Blackburn and $\mathrm{H}$. Whitaker performed statistical analyses. C. Warren-Gash obtained funding and drafted the paper. All authors approved the final manuscript version.

\section{References}

1 Wang H, Naghavi M, Allen C, et al. Global, regional, and national life expectancy, all-cause mortality, and cause-specific mortality for 249 causes of death, 1980-2015: a systematic analysis for the Global Burden of Disease Study 2015. Lancet 2016; 388: 1459-1544.

2 World Health Organization. The top 10 causes of death. www.who.int/mediacentre/factsheets/fs $310 / \mathrm{en} / \mathrm{index} . \mathrm{html}$ Date last accessed: July 20, 2017. Date last updated: January, 2017.

3 Barnes M, Heywood AE, Mahimbo A, et al. Acute myocardial infarction and influenza: a meta-analysis of case-control studies. Heart 2015; 101: 1738-1747.

4 Warren-Gash C, Smeeth L, Hayward AC. Influenza as a trigger for acute myocardial infarction or death from cardiovascular disease: a systematic review. Lancet Infect Dis 2009; 9: 601-610.

5 Corrales-Medina VF, Suh KN, Rose G, et al. Cardiac complications in patients with community-acquired pneumonia: a systematic review and meta-analysis of observational studies. PLoS Med 2011; 8: e1001048.

6 Huijskens EGW, van Erkel AJM, Palmen FMH, et al. Viral and bacterial aetiology of community-acquired pneumonia in adults. Influenza Other Respir Viruses 2013; 7: 567-573. 
7 Hirama T. A Real-time PCR-based diagnostic test for organisms in respiratory tract infection. 2016. www. intechopen.com/books/polymerase-chain-reaction-for-biomedical-applications/a-real-time-pcr-based-diagnostic-testfor-organisms-in-respiratory-tract-infection Date last accessed: May 23, 2017.

8 Udell JA, Zawi R, Bhatt DL, et al. Association between influenza vaccination and cardiovascular outcomes in high-risk patients: a meta-analysis. JAMA 2013; 310: 1711.

9 Simonsen L, Taylor RJ, Viboud C, et al. Mortality benefits of influenza vaccination in elderly people: an ongoing controversy. Lancet Infect Dis 2007; 7: 658-666.

10 Ren S, Newby D, Li SC, et al. Effect of the adult pneumococcal polysaccharide vaccine on cardiovascular disease: a systematic review and meta-analysis. Open Heart 2015; 2: e000247.

11 Vlachopoulos CV, Terentes-Printzios DG, Aznaouridis KA, et al. Association between pneumococcal vaccination and cardiovascular outcomes: a systematic review and meta-analysis of cohort studies. Eur J Prev Cardiol 2015; 22: 1185-1199.

12 Ison MG, Campbell V, Rembold C, et al. Cardiac findings during uncomplicated acute influenza in ambulatory adults. Clin Infect Dis 2005; 40: 415-422.

13 Reyes LF, Restrepo MI, Hinojosa CA, et al. Severe pneumococcal pneumonia causes acute cardiac toxicity and subsequent cardiac remodeling. Am J Respir Crit Care Med 2017; 196: 609-620.

14 Singanayagam A, Singanayagam A, Elder DHJ, et al. Is community-acquired pneumonia an independent risk factor for cardiovascular disease? Eur Respir J 2012; 39: 187-196.

15 Petersen I, Douglas I, Whitaker H. Self controlled case series methods: an alternative to standard epidemiological study designs. BMJ 2016; 354: i4515.

16 Whitaker HJ, Farrington CP, Spiessens B, et al. Tutorial in biostatistics: the self-controlled case series method. Stat Med 2006; 25: 1768-1797.

17 Public Health England. Surveillance of influenza and other respiratory viruses in the United Kingdom: winter 2014 to 2015. Report 2015046. 2015. www.gov.uk/government/uploads/system/uploads/attachment_data/file/ 429617/Annualreport_March2015_ver4.pdf Date last accessed: July 20, 2017.

18 Yang L, Ma S, Chen PY, et al. Influenza associated mortality in the subtropics and tropics: results from three Asian cities. Vaccine 2011; 29: 8909-8914.

19 Green HK, Andrews N, Fleming D, et al. Mortality attributable to influenza in England and Wales prior to, during and after the 2009 pandemic. PLoS One 2013; 8: e79360.

20 Hung IFN, Zhang AJ, To KKW, et al. Unexpectedly higher morbidity and mortality of hospitalized elderly patients associated with rhinovirus compared with influenza virus respiratory tract infection. Int J Mol Sci 2017; 18: E259.

21 Widmer K, Zhu Y, Williams JV, et al. Rates of hospitalizations for respiratory syncytial virus, human metapneumovirus, and influenza virus in older adults. J Infect Dis 2012; 206: 56-62.

22 van Asten L, van den Wijngaard C, van Pelt W, et al. Mortality attributable to 9 common infections: significant effect of influenza A, respiratory syncytial virus, influenza B, norovirus, and parainfluenza in elderly persons. J Infect Dis 2012; 206: 628-639.

23 Lee N, Lui GCY, Wong KT, et al. High morbidity and mortality in adults hospitalized for respiratory syncytial virus infections. Clin Infect Dis 2013; 57: 1069-1077.

24 Blackburn R, Hayward AC, Pebody R, et al. Laboratory-confirmed respiratory infections as predictors of hospital admission for myocardial infarction and stroke: time-series analysis of English data for 2004-2015. Clin Infect Dis 2018 ; in press [https://doi.org/10.1093/cid/cix1144]

25 Smeeth L, Thomas SL, Hall AJ, et al. Risk of myocardial infarction and stroke after acute infection or vaccination. N Engl J Med 2004; 351: 2611-2618.

26 Warren-Gash C, Hayward AC, Hemingway H, et al. Influenza infection and risk of acute myocardial infarction in England and Wales: a CALIBER self-controlled case series study. J Infect Dis 2012; 206: 1652-1659.

27 Public Health England. Influenza immunisation programme for England: GP patient groups data collection survey season 2015-2016. Report 2016087. 2016. www.gov.uk/government/uploads/system/uploads/attachment_data/file/ 544552/Seasonal_flu_GP_patient_groups_annual_report_2015_2016.pdf Date last accessed: June 21, 2017.

28 Ren S, Hure A, Peel R, et al. Rationale and design of a randomized controlled trial of pneumococcal polysaccharide vaccine for prevention of cardiovascular events: the Australian Study for the Prevention through Immunization of Cardiovascular Events (AUSPICE). Am Heart J 2016; 177: 58-65.

29 Wen Y-C, Hsiao F-Y, Chan KA, et al. Acute respiratory infection and use of nonsteroidal anti-inflammatory drugs on risk of acute myocardial infarction: a nationwide case-crossover study. J Infect Dis 2017; 215: 503-509. 